\title{
Beetles of the superfamily Hydrophiloidea of Kemerovo Area
}

\section{Жесткокрылые надсемейства Нydrophiloidea Кемеровской области}

\author{
S.V. Litovkin ${ }^{1}$, D.A. Efimov ${ }^{2}$ \\ С.В. Аитовкин ${ }^{1}$, А.А. Ефимов ${ }^{2}$
}

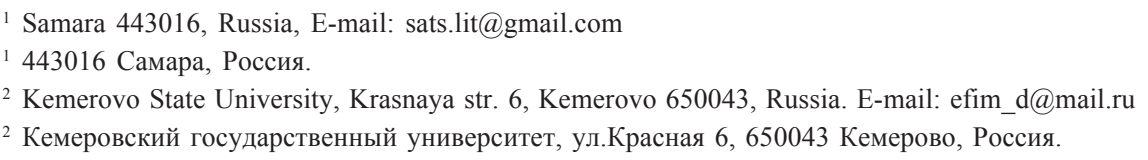

KEY WORDS: Coleoptera, Hydrophiloidea, fauna, new records, Kemerovo Area, Siberia.

КЛЮЧЕВЫЕ СЛОВА: Coleoptera, Hydrophiloidea, фауна, новые находки, Кемеровская область, Сибирь.

ABSTRACT. An annotated checklist of beetles of the superfamily Hydrophiloidea of Kemerovo Area is given. It includes 55 species of three families: Helophoridae (11 species), Hydrochidae (3) and Hydrophilidae (41). Helophorus discrepans Rey, 1885 and Cryptopleurum crenatum (Kugelann, 1794) are recorded for the first time for the Asian part of Russia. Helophorus aspericollis Angus, 1973 and Enochrus melanocephalus (Olivier, 1792) are recorded for the first time for the Western Siberia. Occurrence of species Cercyon ustulatus (Preyssler, 1790) in Western Siberia is confirmed. A total of 31 species are recorded for the first time in Kemerovo Area.

РЕЗЮМЕ. Приведён аннотированный список жесткокрылых надсемейства Hydrophiloidea Кемеровской области. Он включает 55 видов из трёх семейств: Helophoridae (11 видов), Hydrochidae (3) и Hydrophilidae (41 вид). Helophorus discrepans Rey, 1885 и Cryptopleurum crenatum (Kugelann, 1794) впервые приводятся для азиатской части России. Helophorus aspericollis Angus, 1973 и Enochrus melanocephalus (Olivier, 1792) впервые указываются для Западной Сибири. Подтверждено обитание в Сибири Cercyon ustulatus (Preyssler, 1790). В целом, 31 вид впервые приводится для Кемеровской области.

\section{Introduction}

Hydrophyloid line of the superfamily Hydrophiloidea (Hydrophiloidea s.str.) includes six families: Helophoridae, Georissidae, Epimetopidae, Hydrochidae, Spercheidae and Hydrophilidae and more than 3500 recent species [Hansen, 1999; Short, 2017]. Five families, except Epimetopidae, and no less than 210 species are known in the territory of Russia. In Western Siberia, only families Helophoridae, Georissidae, Hydrochidae and Hydrophilidae were reliably recorded [Fikáček et al., 2015; Przewoźny, 2019].

The fauna of Hydrophiloidea of Kemerovo Area is poorly studied. There is only one special publication dedicated to the region's hydrophiloid beetles [Efimov, 2010]. A few information about representatives of the region's superfamily is also contained in a number of faunistic works [Efimov, Zinchenko, 2015; Ryndevich, 2003a, 2017; Hebauer, Ryndevich, 2005], taxonomic revisions and review [Ryndevich, 2003b, 2004; Ryndevich et al., 2017]. In total the listed papers cite data on one species of Helophoridae, two species of Hydrochidae, and 24 species of Hydrophilidae.

This article includes new findings of Hydrophiloid beetles that expand knowledge of the fauna of aquatic beetles as of Kemerovo Area and Western Siberia, and the entire Asian part of Russia.

\section{Material and methods}

The present study is based mainly on the material which has been collected by the second author, and has been provided by collegues.

The main part of the material was determined by the first author, part of Helophoridae was determined by A.A. Prokin and A.S. Sazhnev (Borok) and part of Hydrophilidae and Helophoridae by V.K. Zinchenko (Novosibirsk).

Photographs of beetles and their details were taken with a Nikon D3200 camera, equipped with a microscope objective LOMO PLAN 3.5×0.10. Photographs of genitalia were taken by Canon PowerShot A640 camera, attached with a LOMO Biolam R-17 compound microscope. Stacking and processing of photos carried

How to cite this article: Litovkin S.V., Efimov D.A. 2020. Beetles of the superfamily Hydrophiloidea of Kemerovo Area // Russian Entomol. J. Vol.29. No.1. P.61-68. doi: 10.15298/rusentj.29.1.08 
out in programs Helicon Focus 5.3 and Adobe Photoshop CS3.The list of all species of Hydrophiloidea noted for the Kemerovo Area with reference to literature, new finds, general distribution and the necessary comments is given below.

A significant part of the material was collected in the vicinity of the biological station of KemSU "Azhendarovo", Krapivino district, $8 \mathrm{~km}$ SSW of Saltymakovo village $\sim 54^{\circ} 45^{\prime} 18^{\prime \prime} \mathrm{N} 87^{\circ} 01^{\prime} 43^{\prime \prime} \mathrm{E}$. In the text this locality is briefly indicated as "Azhendarovo".

Additionally some frequently mentioned names of the collectors are abbreviated as follows: F.A. Budaev (FB), D.A. Efimov (DE), A.V. Korshunov (AK).

Studied material is deposited in private collections of authors, unless otherwise indicated, and partly in the collections of Institute of Systematics and Ecology of Animals (ISEA, Novosibirsk, Russia) and in Papanin Institute for Biology of Inland Waters Russian Academy of Sciences (IBIW RAS, Borok, Russia).

Species new to the Kemerovo Area are indicated by one asterisk $(*)$, species new for the Western Siberia are indicated by double asterisk $(* *)$. The species wrong determined earlier are excluded from through numbering. The names of the species are given in a systematic order, but without specifying taxa of a rank lower than the family.

\section{List of taxa}

\section{Family HELOPHORIDAE Leach, 1815 \\ 1. Helophorus (Rhopalohelophorus) aspericollis Angus, 1973**}

Fig. 1.

MATERIAL EXAMINED. Kemerovskiy Distr., Krekovo, $55^{\circ}$ $31^{\prime} \mathrm{N} 85^{\circ} 52^{\prime} \mathrm{E}$, 29.VII.2009, 6.VII.2013, DE (8 exs.); Izhmorskiy Distr., Tunda, pond, 18.VIII.2018, DE (1 ex.).

DISTRIBUTION. Eastern Siberia, Russian Far East, Kazakhstan, Mongolia, Western China [Fikáček et al., 2015; Przewoźny, 2019]. New for Kemerovo Area and Western Siberia.

\section{Helophorus (Rhopalohelophorus) auricollis Eschscholtz, 1822}

REFERENCES. Ryndevich [2003a].

MATERIAL EXAMINED. No specimens examined by authors.

DISTRIBUTION. Western and Eastern Siberia, Russian Far East [Ryndevich, 2003a; Hansen, 2004], Canada, USA [Hansen, 1999].

\section{Helophorus (Rhopalohelophorus) barbarae Angus, 1985*}

MATERIAL EXAMINED . Krapivinskiy Distr., near "Azhendarovo", 28.V.-3.VI.2014, FB (1 ex., ISEA), A. Prokin and A. Sazhnev det.

DISTRIBUTION. South part of European Russia, Western Siberia, Kazakhstan [Fikáček et al., 2015; Przewoźny, 2019]. New for Kemerovo Area.

\section{Helophorus (Rhopalohelophorus) croaticus Kuwert, 1886*}

MATERIAL EXAMINED. Krapivinskiy Distr., near "Azhendarovo", 28.V-3.VI.2014, FB (3 exs., ISEA), A. Prokin and A. Sazhnev det.
DISTRIBUTION. Europe, Western and Eastern Siberia, Turkey [Fikáček et al., 2015; Przewoźny, 2019]. New for Kemerovo Area.

\section{Helophorus (Rhopalohelophorus) discrepans Rey, 1885**}

MATERIAL EXAMINED. Krapivinskiy Distr., near “Azhendarovo", 2.V.2014, 28.V.-3.VI.2014, FB (2 exs., ISEA), A. Prokin and A. Sazhnev det.

DISTRIBUTION. Europe, Morocco, Turkey, Iran [Fikáček et al., 2015; Przewoźny, 2019]. New for Kemerovo Area and Asian part of Russia.

\section{Helophorus (Rhopalohelophorus) granularis (Linnaeus, 1760)*}

MATERIAL EXAMINED. Kemerovskiy Distr., Krekovo, 553 $1^{\prime} \mathrm{N} 85^{\circ} 52^{\prime} \mathrm{E}, 23$.VII.2009, 29.VII.2009, DE (2 exs.); Krapivin-

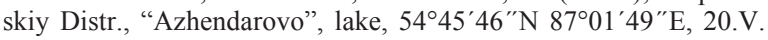
2012, DE (1 ex.); Yayskiy Distr., Ishim, shore of Yaya River, 10.VII.2012, DE (2 exs.); Tyazhynskiy Distr., Kamyshlovka, 55 $58^{\prime} \mathrm{N}$ $88^{\circ} 32^{\prime} \mathrm{E}$, bog, 10.VI.2015, DE (3 exs.); Pritomskiy, pump house, 12.V.2015, FB (1 ex., IBIW), A. Prokin and A. Sazhnev det.; Promyshlennovskiy Distr., Krasninskoe, 12.V.2015, FB (1 ex., IBIW), A. Prokin and A. Sazhnev det.; Kemerovskiy Distr., Pod'yakovo, Bol'shaya Podikova River, 9.VII.2015, DE (3 exs.); Kemerovo, FPK, lake, 55²1'61.0"N 87²13'66.1'”, 26.V.2016, FB (1 ex., IBIW), A. Prokin and A. Sazhnev det.; Izhmorskiy Distr., Novyy Svet, shore of Zolotoy Kitat river, 5546.160'N 86³9.343'E, 11.VII.2018, DE (1 ex.); Izhmorskiy Distr., Tunda, pond, 18.VIII. 2018, DE (3 exs.).

DISTRIBUTION. Europe, Western and Eastern Siberia, Turkey [Fikáček et al., 2015; Przewoźny, 2019]. New for Kemerovo Area.

\section{Helophorus (Rhopalohelophorus) lapponicus Thomson, 1853*}

MATERIAL EXAMINED. Kemerovskiy Distr., Elykaevo, $55^{\circ}$ $20^{\prime} 73^{\prime \prime} \mathrm{N} 86^{\circ} 07^{\prime} 26^{\prime \prime} \mathrm{E}, 21 . \mathrm{V} .2016$, FB (1 ex., IBIW), A. Prokin and A. Sazhnev det.

DISTRIBUTION. Eastern Europe, Western and Eastern Siberia, Russian Far East, Middle East, Central Asia, Turkey [Fikáček et al., 2015; Przewoźny, 2019]. New for Kemerovo Area.

\section{Helophorus (Rhopalohelophorus) nanus Sturm, 1836*}

MATERIAL EXAMINED. Kemerovo, Leninskiy Distr., 7.VI.2009, AK (1 ex.); Krapivinskiy Distr., "Azhendarovo", lake, 544' $46^{\prime \prime} \mathrm{N} 87^{\circ} 01^{\prime} 49^{\prime \prime} \mathrm{E}, 20 . \mathrm{V} .2012$, DE (1 ex.); Kemerovo, Kirovskiy Distr., puddle, 2.VI.2016, FB (2 exs., IBIW), A. Prokin and A. Sazhnev det.

DISTRIBUTION. Europe, Western and Eastern Siberia, Russian Far East, Turkey, Northern China [Fikáček et al., 2015; Przewoźny, 2019]. New for Kemerovo Area.

\section{Helophorus (Rhopalohelophorus) paraminutus Angus, 1986*}

MATERIAL EXAMINED. Krapivinskiy Distr., near “Azhendarovo", 24.VII.2009, AK (2 exs.).

DISTRIBUTION. Central and Eastern Europe, Western and Eastern Siberia, Turkey [Fikáček et al., 2015; Przewoźny, 2019]. New for Kemerovo Area.

\section{Helophorus (Rhopalohelophorus) redtenbacheri Kuwert, 1886*}

MATERIAL EXAMINED. Krapivinskiy Distr., near “Azhendarovo", 28.V-03.VI.2014, FB (1 ex.), V. Zinchenko det 
DISTRIBUTION. Central and Eastern Europe, Western Siberia, Kazakhstan [Fikáček et al., 2015; Przewoźny, 2019]. New for Kemerovo Area.

\section{Helophorus (Rhopalohelophorus) strigifrons Thomson, 1868*}

MATERIAL EXAMINED. Krapivinskiy Distr., near "Azhendarovo", 24.VII.2009, AK (1 ex.); Krapivinskiy Distr., near Sarap-

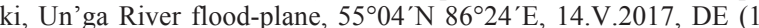
ex.); Yayskiy Distr., Beket, $56^{\circ} 22^{\prime} \mathrm{N} 86^{\circ} 28^{\prime} \mathrm{E}$, water's edge, 15.VI 2018, DE (1 ex.), V. Zinchenko det.

DISTRIBUTION. Europe, Western and Eastern Siberia, Turkey [Fikáček et al., 2015; Przewoźny, 2019]. New for Kemerovo Area.

\section{Family HYDROCHIDAE Thomson, 1859}

12. Hydrochus brevis (Herbst, 1793)

REFERENCES. Efimov, Zinchenko [2015].

MATERIAL EXAMINED. Tyazhynskiy Distr., Kamyshlovka, $55^{\circ} 58^{\prime} \mathrm{N} 88^{\circ} 32^{\prime} \mathrm{E}$, bog, 10.VI.2015, DE (1 ex.).

DISTRIBUTION. Europe, Western and Eastern Siberia, Turkey [Ryndevich, 2014; Efimov, Zinchenko, 2015; Fikáček et al., 2015].

13. Hydrochus elongatus (Schaller, 1783)

REFERENCES. Efimov, Zinchenko [2015].

MATERIAL EXAMINED. Yayskiy Distr., Beket, 56 $22^{\prime} \mathrm{N}$ $86^{\circ} 28^{\prime} \mathrm{E}$, water's edge, 15.VI.2018, DE (1 ex.).
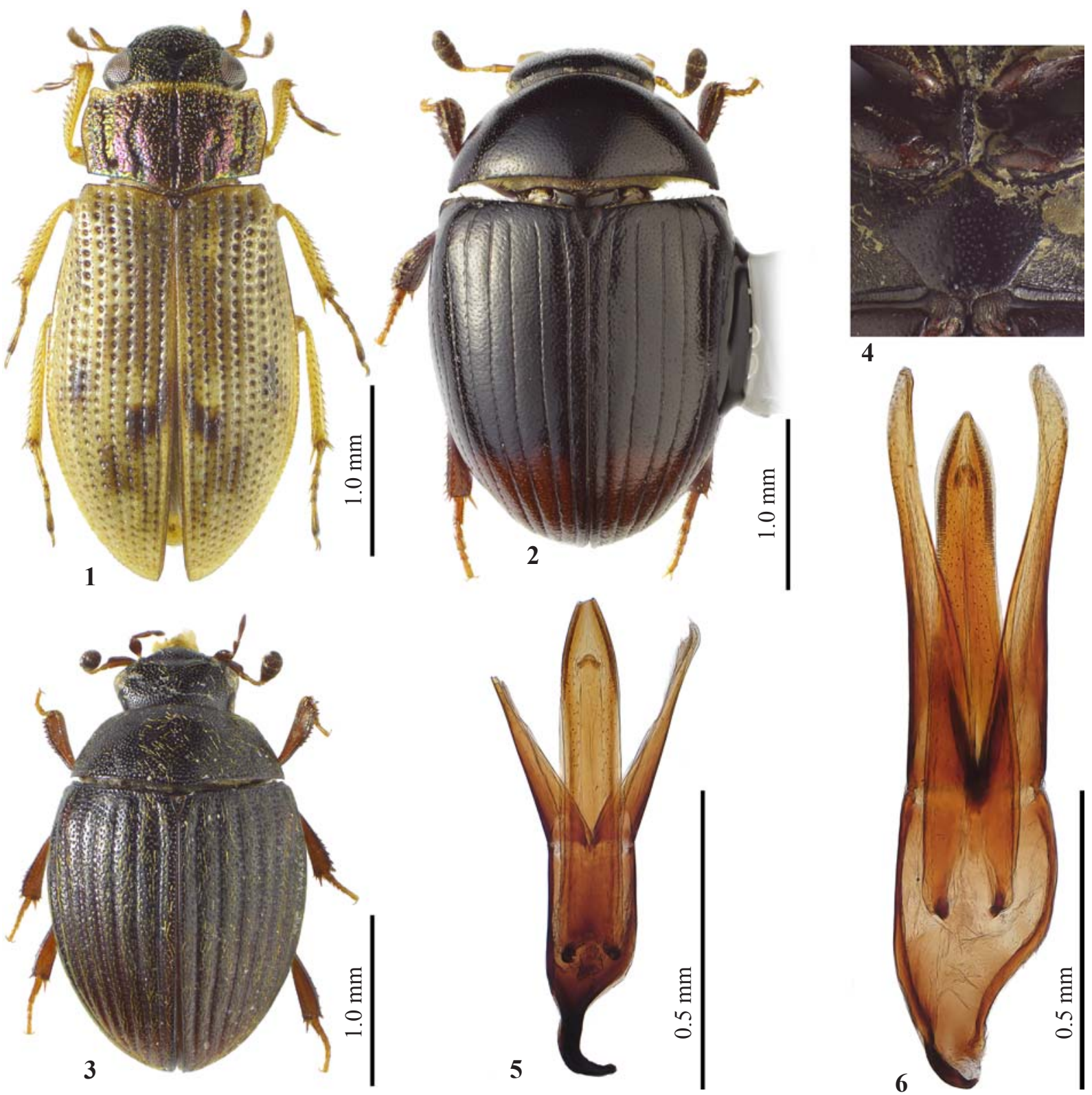

3

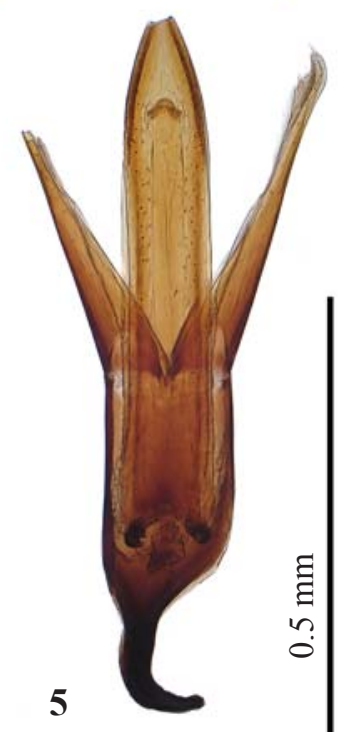

Figs 1-6. Hydrophiloidea of Kemerovo Area: 1 - Helophorus aspericollis; 2, 4, 6 - Cercyon ustulatus; 3, 5 - Cryptopleurum crenatum; $1-3$ - general view; 4 - meso- and metathorax; 5-6 - aedeagi.

Рис. 1-6. Hydrophiloidea Кемеровской области: 1 - Helophorus aspericollis; 2, 4, 6 - Cercyon ustulatus; 3, 5 - Cryptopleurum crenatum; 1-3 - общий вид; 4 - средне- и заднегрудь; 5-6 - гениталии самцов. 
DISTRIBUTION. Europe, Western and Eastern Siberia, Central Asia, Turkey [Fikáček et al., 2015; Przewoźny, 2019].

\section{Hydrochus ignicollis Motschulsky, 1860*}

MATERIAL EXAMINED. Izhmorskiy Distr., Novyy Svet, shore

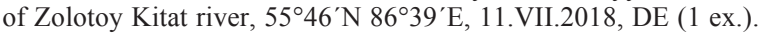

DISTRIBUTION. Europe, Western Siberia, Turkey [Fikáček et al., 2015; Przewoźny, 2019]. New for Kemerovo Area.

\section{Family HYDROPHILIDAE Latreille, 1802}

\section{Berosus (Berosus) luridus Linnaeus, 1760*}

MATERIAL EXAMINED. Leninsk-Kuznetskiy Distr., vicinity of Podgornoe, $54^{\circ} 28^{\prime} 10^{\prime \prime} \mathrm{N} 86^{\circ} 00^{\prime} 26^{\prime \prime} \mathrm{E}$, pit, 1.VI.2017, FB (1 ex.)

DISTRIBUTION. Europe, Western Siberia, Turkey, Iran [Fikáček et al., 2015; Przewoźny, 2019]. New for Kemerovo Area.

REMARKS. Probably the most eastern finding of the species. All reports for Western Siberia [Fikáček et al., 2015] refers to the Chelyabinsk Area [Prokin et al., 2008]. Reports of the species for the Irkutsk Area [Berlov, 1978] requires verification.

\section{Berosus (Berosus)}

signaticollis (Charpentier, 1825)

REFERENCES. Efimov [2010].

MATERIAL EXAMINED. Kemerovskiy Distr., Starochervovo, $55^{\circ} 10^{\prime} \mathrm{N} 86^{\circ} 22^{\prime} \mathrm{E}$, 19.IV.2014, FB (1 ex.), V. Zinchenko det.

DISTRIBUTION. Western Palaearctic [Fikáček et al., 2015; Przewoźny, 2019].

\section{Berosus (Enoplurus) spinosus (Steven, 1808)}

REFERENCES. Efimov, Zinchenko [2015].

MATERIAL EXAMINED. No specimens examined by authors DISTRIBUTION. Western Palaearctic, Mongolia, China [Fikáček et al., 2015; Przewoźny, 2019].

\section{Laccobius (Dimorpholaccobius) bipunctatus (Fabricius, 1775)*}

MATERIAL EXAMINED. Kemerovskiy Distr., Zhuravlyovo, $55^{\circ} 08.135^{\prime} \mathrm{N} 86^{\circ} 20.058^{\prime} \mathrm{E}, 21 . \mathrm{V} .2016$, FB (3 exs.).

DISTRIBUTION. Western Palaearctic [Fikáček et al., 2015; Przewoźny, 2019]. New for Kemerovo Area.

\section{Laccobius (Laccobius) minutus (Linnaeus, 1758)*}

MATERIAL EXAMINED. Kemerovo, small pool, 16.VIII.2009, DE (1 ex.); Kemerovo, water's edge, 9.V.2009, V. Polevod leg. (1 ex.) Kuznetsk Alatau, cordon "Verkhnyaya Ters", Verkhnyaya Ters' River, under stones, $54^{\circ} 10^{\prime} \mathrm{N} 88^{\circ} 07^{\prime} \mathrm{E}$, 4.VII.2009, AK (1 ex.); Yayskiy Distr., Ishim, shore of Yaya River, 10.VII.2012, DE (12 exs.); Krapivinskiy Distr., near "Azhendarovo", 20.V.2012, 28.V.-3.VI.2014, 28.VII.2017, FB, DE (8 exs.); Kemerovskiy Distr., Mozzhukha, 55²5’ $85^{\circ} 56^{\prime}$ E, 9.VI.2013, DE (1 ex.); Promyshlennovskiy Distr., Krasninskoe, 12.V.2015, FB (1 ex.); Kemerovskiy Distr., Starochervovo, $55^{\circ} 10^{\prime} \mathrm{N} 86^{\circ} 22^{\prime} \mathrm{E}, 22 . \mathrm{VI} .2015$, FB (10 exs.); Kemerovskiy Distr., Pod'yakovo, bog, 9.VII.2015, DE (1 ex.); Kemerovskiy Distr., Zhuravlyovo, $55^{\circ} 08^{\prime} \mathrm{N} 86^{\circ} 20^{\prime}$ E, 21.V.2016, FB (2 exs.); Leninsk-Kuznetskiy Distr., vicinity of Podgornoe, $54^{\circ} 28^{\prime} 10^{\prime \prime} \mathrm{N} 86^{\circ} 00^{\prime} 26^{\prime \prime} \mathrm{E}, 1 . \mathrm{V} .2017$, 1.VI.2017, 15.VIII.2017, FB (20 exs.); Krapivinskiy Distr., near Ban-

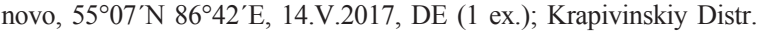

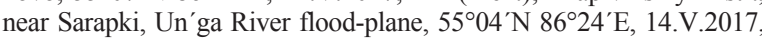
DE (1 ex.); Kemerovskiy Distr., Krekovo, $55^{\circ} 31^{\prime} \mathrm{N} 85^{\circ} 52^{\prime} \mathrm{E}$, pond, 20.V.2017, DE (1 ex.); Kemerovo, Krasnoe Lake, 25.VII.2017, FB (1 ex.); Krapivinskiy Distr., Taradanovo, river, $54^{\circ} 45^{\prime} \mathrm{N} 86^{\circ} 40^{\prime} \mathrm{E}$, 13.VIII.2017, DE (1 ex,); Izhmorskiy Distr., Novyy Svet, shore of Zolotoy Kitat River, 55 $46^{\prime} \mathrm{N} 86^{\circ} 39^{\prime} \mathrm{E}, 11 . \mathrm{VII} .2018$, DE (1 ex.).

DISTRIBUTION. Europe, Northern and Central Asia, Turkey, Mongolia, China [Fikáček et al., 2015; Przewoźny, 2019]. New for Kemerovo Area.

\section{Laccobius (Laccobius) colon (Stephens, 1829)*}

MATERIAL EXAMINED. Krapivinskiy Distr. "Azhendarovo", lake, 544ㄴ $46^{\prime \prime N} 87^{\circ} 01^{\prime} 49^{\prime \prime}$ E, 21-23.V.2011, 20.V.2012, AK (12 exs.) Izhmorskiy Distr., Tunda, pond, 18.VIII.2018, DE (1 ex.).

DISTRIBUTION. Europe, Northern and Central Asia, Mongolia, China [Fikáček et al., 2015; Przewoźny, 2019]. New for Kemerovo Area.

\section{Hydrobius fuscipes (Linnaeus, 1758)}

REFERENCES. Efimov [2010].

MATERIAL EXAMINED. Kemerovo, at light, 1.VI.1998, AK (1 ex.), V. Zinchenko det.; Gornaya Shoriya, Tashtagol'skiy Distr., 4 km N Sheregesha, Zelenaya Mt., 29-31.VII.1999, DE (1 ex.), V. Zinchenko det.; Kemerovskiy Distr., near Sukhaya Rechka, 31.VII.2000, D. Sushchev (1 ex.), V. Zinchenko det.; Gornaya Shoriya, Mustag Mt., 8.VII.2001, DE (1 ex.), V. Zinchenko det.; Kemerovskiy Distr., Osinovka, at light, 16.VIII.2002, AK (1 ex.), V. Zinchenko det.; Yashkinskiy Distr., Pashkovo, at light, 17.VI.2003, AK (1 ex.), V. Zinchenko det.; Krapivinskiy Distr., near "Azhendarovo", 20-28.V.2012,28.V.-3.VI.2014,02.V.2014, FB, AK (30 exs.), V. Zinchenko det.; Kemerovo, 3.IX.2013, FB (1 ex.), V. Zinchenko det.; Leninsk-Kuznetskiy Distr., near Shabanovo, mouth of Sukhoy creek, $54^{\circ} 40^{\prime} \mathrm{N} 85^{\circ} 32^{\prime} \mathrm{E}, 25$.VII.2014, DE (1 ex.); Kemerovskiy Distr., Starochervovo, $55^{\circ} 10^{\prime} \mathrm{N} 86^{\circ} 22^{\prime} \mathrm{E}, 22$.VI.2015, FB (3 exs.); Kemerovskiy Distr., Elykaevo, $55^{\circ} 20^{\prime} \mathrm{N} 86^{\circ} 07^{\prime} \mathrm{E}, 21 . \mathrm{V} .2016$, FB (2 exs.); Leninsk-Kuznetskiy Distr., vicinity of Podgornoe, $54^{\circ} 28^{\prime} 10^{\prime \prime} \mathrm{N}$ $86^{\circ} 00^{\prime} 26^{\prime \prime} \mathrm{E}, 1 . \mathrm{V} .2017$, FB (3 exs.); Yayskiy Distr., Beket, $56^{\circ} 22^{\prime} \mathrm{N}$ $86^{\circ} 28^{\prime} \mathrm{E}$, in dung near water, 15.VI.2018, DE (1 ex.); Yashkinskiy Distr., near Akatsiya, $55^{\circ} 42^{\prime} \mathrm{N} 85^{\circ} 29^{\prime} \mathrm{E}, 20 . \mathrm{VI} .2018$, DE (4 exs.).

DISTRIBUTION. Holarctic [Hansen, 1999; Fikáček et al., 2015; Przewoźny, 2019].

REMARKS. Currently, Hydrobius fuscipes is considered as a complex of species, the taxonomic composition of which is not completely understood [Fossen et al., 2016]. The material presented here needs further study.

\section{Hydrochara caraboides (Linnaeus, 1758)}

REFERENCES. Efimov [2010].

MATERIAL EXAMINED. Krapivinskiy, 25.VII.2007, T. Platonova leg. (1 ex.); Krapivinskiy Distr., near "Azhendarovo", 10.22.VIII.2010, 20.-28.V.2012, 28.V.-03.VI.2014, DE, FB, AK, A. Kostyunin leg. (6 exs.); vicinity of "Azhendarovo", moth of Bugas River, $54^{\circ} 44^{\prime} \mathrm{N} 87^{\circ} 01^{\prime} \mathrm{E}, 10 .-30 . \mathrm{VII} .2008$, AK (2 exs.); Promyshlennovskiy Distr., Morozovo, pool, 22.VI.2012, DE (1 ex.).

DISTRIBUTION. Europe, Northern Africa, Western and Eastern Siberia, Turkey, Iran [Fikáček et al., 2015; Przewoźny, 2019].

\section{- Hydrochara dichroma (Fairmaire, 1892)}

REMARKS. Records of this species for the Kemerovo Area [Efimov, 2010] is based on a mistakenly signed specimen of Hydrobius fuscipes (L.).

\section{Hydrophilus aterrimus Eschscholtz, 1822*}

MATERIAL EXAMINED. Kemerovskiy Distr., Beregovoy, at night on road, 18.VIII.1999, Yu. Danchenko leg. (1 ex.); Krapivin-

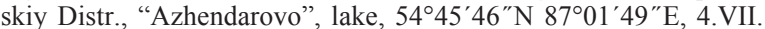
2001, N. Teplova leg. (1 ex.); Kemerovo, near airport, 7.VIII.2003 (2 exs.); Mariinsk, 3.VIII.2004, N. Lobova leg. (1 ex.); Promyshlennaya, 27.VIII.2005, 25.VII.2007, M. Samoshchenko, A. Erin leg. (2 exs.); Yashkinskiy Distr., Yashkino, at light, 06.-7.VIII.2016, V. Akaev leg. (1 ex., coll. V. Akaev).

DISTRIBUTION. Europe except its westernmost parts, Western Siberia, Turkey, Iran, Turkmenistan [Fikáček et al., 2015; Przewoźny, 2019]. New for Kemerovo Area.

REMARKS. See comment for the following species.

- Hydrophilus piceus (Linnaeus, 1758)

REMARKS. All records from the Kemerovo Area previously cited under this name [Efimov, 2010] belong to 
H. aterrimus. The distribution of the species in Western Siberia as a whole requires clarification.

\section{Anacaena lutescens (Stephens, 1829)}

REFERENCES. Ryndevich [2003b]; Efimov [2010].

MATERIAL EXAMINED. Tyazhinskiy Distr., left shore of Uryup River near confluence with Chulym River, oxbow, 1.VIII.2008, N. Teplova leg. (1 ex.); Kuznetskiy Alatau, cordon "Verkhnyaya Ters", Verkhnyaya Ters' River, under stones, $\mathrm{h}=409 \mathrm{~m}$ a.s.l., 04.VII.2009, AK (1 ex.); Krapivinskiy Distr., near "Azhendarovo", 24.VII.2009, 20 28.V.2012, 20.V.2012, 2.V.2014, 28.V.-03.VI.2014, AK, DE, FB 15 exs.); Promyshlennovskiy Distr., Krasninskoe, 12.V.2015, FB (6 exs.);

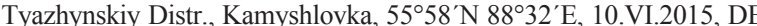
(1 ex.); Kemerovskiy Distr., Pod'yakovo, Bolshaya Podikova River, 09.VII.2015, DE (1 ex.); Kemerovskiy Distr., Starochervovo, $55^{\circ} 10^{\prime} \mathrm{N}$ $86^{\circ} 22^{\prime}$ E, 7.IX.2015, FB (1 ex.); Kemerovo Distr. Zhuravlevo, $55^{\circ} 08^{\prime} \mathrm{N}$ $86^{\circ} 20^{\prime}$ E, 21.V.2016, FB (3 exs.); Kemerovskiy Distr., Elykaevo, $55^{\circ} 20^{\prime} \mathrm{N} 86^{\circ} 07^{\prime} \mathrm{E}$, 21.V.2016, FB (12 exs.); Krapivinskiy Distr., near

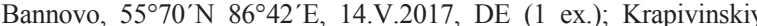
Distr., near Sarapki, Un' ga River flood-plane, $55^{\circ} 04^{\prime} \mathrm{N} 86^{\circ} 24^{\prime} \mathrm{E}, 14 . \mathrm{V}$ 2017, DE (3 exs.); Leninsk-Kuznetskiy Distr., vicinity of Podgornoe, $54^{\circ} 28^{\prime} 10^{\prime \prime} \mathrm{N} 86^{\circ} 00^{\prime} 26^{\prime \prime}$ E, 1.VI.2017, FB (3 exs.); Krapivinskiy Distr. Taradanovo, river, $54^{\circ} 45^{\prime} \mathrm{N} 86^{\circ} 40^{\prime} \mathrm{E} 13 . \mathrm{VIII} .2017$, DE (1 ex.); Yayskiy Distr., Beket, $56^{\circ} 22^{\prime} \mathrm{N} 86^{\circ} 28^{\prime} \mathrm{E}, 15 . \mathrm{VI} .2018$, DE (8 exs.); Yashkinskiy

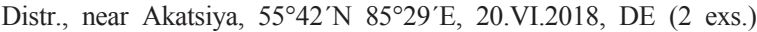

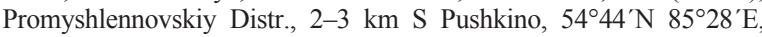
8.VIII.2018, DE (2 exs.).

DISTRIBUTION. Western Palaearctic, Russian Far East [Fikáček et al., 2015; Przewoźny, 2019], Canada, USA [Hansen, 1999].

REMARKS. All 49 studied specimens were females; the sex of another 12 specimens was not determined. This, most likely, indicates the homogeneity of the local population and its reproduction through parthenogenesis. As known, most European populations of $A$. lutescens are also parthenogenetic [Shaarawi, Angus, 1990; Ryndevich, 2003b].

\section{Cymbiodyta marginella (Fabricius, 1792)*}

MATERIAL EXAMINED. Krapivinskiy Distr., "Azhendarovo",

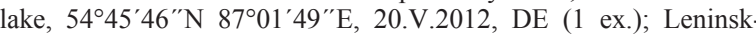
Kuznetskiy Distr., vicinity of Podgornoe, $54^{\circ} 28^{\prime} 10^{\prime \prime} \mathrm{N} 86^{\circ} 00^{\prime} 26^{\prime \prime} \mathrm{E}$ pit, 15.VIII.2017, FB (1 ex.); Kemerovskiy Distr., Elykaevo, $55^{\circ} 20^{\prime} \mathrm{N}$ $86^{\circ} 07^{\prime} \mathrm{E}, 21 . \mathrm{V} .2016$, FB (6 exs.).

DISTRIBUTION. Europe, Turkey, Western and Eastern Siberia, Central Asia, Nepal [Fikáček et al., 2015; Przewoźny, 2019]. New for Kemerovo Area.

\section{Enochrus (Enochrus) \\ melanocephalus (Olivier, 1792)**}

MATERIAL EXAMINED. Kemerovskiy Distr., Starochervovo, $55^{\circ} 10^{\prime} \mathrm{N} 86^{\circ} 22^{\prime} \mathrm{E}, 22$.VI.2015, FB (1 ex.).

DISTRIBUTION. Europe, Northern Africa, Western Asia, Eastern Siberia, Russian Far East, Mongolia, Northern China [Fikáček et al., 2015; Przewoźny, 2019; Prokin et al., 2020]. New for Kemerovo Area and Western Siberia.

\section{Enochrus (Lumetus) bicolor (Fabricius, 1792)*}

MATERIAL EXAMINED. Promyshlennovskiy Distr., Morozovo, water's edge of shallow pool, in water, 22.VI.2012, DE (4 exs.); LeninskKuznetskiy Distr., near Shabanovo, moth of Sukhoy creek, $54^{\circ} 40^{\prime} \mathrm{N}$ 85³2'E, 25.VII.2014, DE (2 exs.); Promyshlennovskiy Distr., Krasninskoe, lake, 12.V.2015, FB (5 exs.); Promyshlennovskiy Distr., 2-3 km S

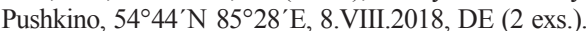

DISTRIBUTION. Palaearctic [Fikáček et al., 2015; Przewoźny, 2019]. New for Kemerovo Area.

\section{Enochrus (Lumetus)}

fuscipennis (Thomson, 1884)*

MATERIAL EXAMINED. Kemerovskiy Distr., Sukhovo, shore of Tom' River, 19.VII.2013, DE (1 ex.); Leninsk-Kuznetskiy Distr.,

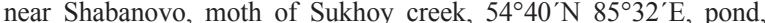
25.VII.2014, DE (1 ex.); Kemerovskiy Distr., Zhuravlevo, 21.V.2016, $55^{\circ} 08^{\prime} \mathrm{N} 86^{\circ} 20^{\prime} \mathrm{E}, \mathrm{FB}$ (1 ex.); Kemerovskiy Distr., Elykaevo, $55^{\circ} 20^{\prime} \mathrm{N}$ $86^{\circ} 07^{\prime} \mathrm{E}, 21 . \mathrm{V} .2016$, FB (29 exs.).

DISTRIBUTION. Europe, Northern Africa, Western and Central Asia, Western and Eastern Siberia, China [Fikáček et al., 2015; Przewoźny, 2019]. New for Kemerovo Area.

\section{Enochrus (Lumetus) ochropterus (Marsham, 1802)*}

MATERIAL EXAMINED. Tyazhinskiy Distr., left shore of Uryup River near confluence with Chulym River, 01.VIII.2008, N. Teplova leg. (1 ex.); Krapivinskiy Distr., near "Azhendarovo", 02.V.2014, FB (2 exs.), V. Zinchenko det.; Kemerovskiy Distr.,

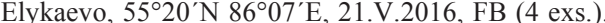

DISTRIBUTION. Europe, Turkey, Western and Eastern Siberia, Iran [Fikáček et al., 2015; Przewoźny, 2019]. New for Kemerovo Area.

\section{Enochrus (Lumetus)} quadripunctatus (Herbst, 1797)*

MATERIAL EXAMINED. Yashinskiy Distr., Ishim, shore of Yaya River, 10.VII.2012, DE (1 ex.); Kemerovskiy Distr., Osinovka, at light, 16.VIII.2012, AK(1 ex.); Leninsk-Kuznetskiy Distr., near Shabanovo, moth of Sukhoy creek, $54^{\circ} 40^{\prime} \mathrm{N} 85^{\circ} 32^{\prime} \mathrm{E}, 25$.VII.

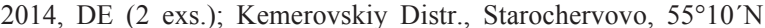
$86^{\circ} 22^{\prime}$ E, 22.VI.2015, FB (1 ex.); Kemerovskiy Distr., Elykaevo, $55^{\circ} 20^{\prime} \mathrm{N} 86^{\circ} 07^{\prime} \mathrm{E}, 21 . \mathrm{V} .2016$, FB (17 exs.); Kemerovo, Krasnoe Lake, 25.VII.2017, 21.IX.2017, FB (4 exs.); Promyshlennovskiy

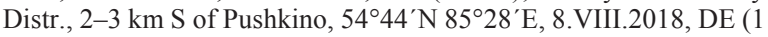
ex.).

DISTRIBUTION. Europe, Turkey, Iran, Northern and Central Asia, Mongolia, China [Fikáček et al., 2015; Przewoźny, 2019]. New for Kemerovo Area.

\section{Enochrus (Lumetus) testaceus (Fabricius, 1801)*}

MATERIAL EXAMINED. Leninsk-Kuznetskiy Distr., vicinity of Podgornoe, $54^{\circ} 28^{\prime} 10^{\prime \prime} \mathrm{N} 86^{\circ} 00^{\prime} 26^{\prime \prime} \mathrm{E}$, pit, 1.VI.2017, 15.VIII.2017, FB (2 exs.); Krapivinskiy Distr., vicinity of Zelenogorskiy, lake, $55^{\circ} 01^{\prime} \mathrm{N} 87^{\circ} 03^{\prime} \mathrm{E}, 9 . \mathrm{VII} .2017$, DE (1 ex.).

DISTRIBUTION. Europe, Northern Africa, Turkey, Iran, Kazakhstan, Western China, Northern Asia, Mongolia, Japan [Fikáček et al., 2015; Przewoźny, 2019; Prokin et al., 2019]. New for Kemerovo Area.

\section{Enochrus (Methydrus) affinis (Thunberg, 1794)*}

MATERIAL EXAMINED. Promyshlennovskiy Distr., Morozovo, 22.VI.2012, DE (1 ex.); Yayskiy Distr., Ishim, shore of Yaya River, 10.VII.2012, DE (1 ex.); Krapivinskiy Distr., near "Azhendarovo", 20-28.V.2012, 28.V.-03.VI.2014, AK, FB (3 exs.), V. Zinchenko det.; Leninsk-Kuznetskiy Distr., near Shabanovo, moth of Sukhoy creek, $54^{\circ} 40^{\prime} \mathrm{N} 85^{\circ} 32^{\prime} \mathrm{E}$, 25.VII.2014, DE (1 ex.); Starochervovo, 22.VI.2015, 7.IX.2015, FB (5 exs.); Kemerovskiy Distr., Elykaevo, $55^{\circ} 20^{\prime} \mathrm{N} 86^{\circ} 07^{\prime} \mathrm{E}, 21 . \mathrm{V} .2016$, FB (3 exs.); Kemerovskiy Distr., Krekovo, $55^{\circ} 31^{\prime} \mathrm{N} 85^{\circ} 52^{\prime} \mathrm{E}$, pond, 20.V.2017, DE (1 ex.); Leninsk-Kuznetskiy Distr., vicinity of Podgornoe, $54^{\circ} 28^{\prime} 10^{\prime \prime} \mathrm{N}$ $86^{\circ} 00^{\prime} 26^{\prime \prime}$ E, 1.VI.2017, 15.VIII.2017, FB (2 exs.); Kemerovo, Krasnoe Lake, 25.VII.2017, FB (3 exs.); Yayskiy Distr., Beket, $56^{\circ} 22^{\prime} \mathrm{N}$ $86^{\circ} 28^{\prime} \mathrm{E}, 15$.VI.2018, DE (2 exs.); Yashkinskiy Distr., near Akatsiya, $55^{\circ} 42^{\prime} \mathrm{N} 85^{\circ} 29^{\prime} \mathrm{E}, 20 . \mathrm{VI} .2018$, DE (1 ex.).

DISTRIBUTION. Europe, Northern Africa, Iran, Kazakhstan, Northern Asia, China, Japan [Fikáček et al., 2015; Przewoźny, 2019]. New for Kemerovo Area.

\section{Enochrus (Methydrus)} coarctatus (Gredler, 1863)*

MATERIAL EXAMINED. Krapivinskiy Distr., near "Azhendarovo", 20.V.2012, 20.-28.V.2012, 2.V.2014, DE, AK, FB (13 exs.); Yayskiy Distr., Beket, $56^{\circ} 22^{\prime} \mathrm{N} 86^{\circ} 28^{\prime} \mathrm{E}, 15 . \mathrm{VI} .2018$, DE (1 ex.). 
DISTRIBUTION. Europe, Turkey, Western Siberia, Russian Far East, Iran, Mongolia [Fikáček et al., 2015; Przewoźny, 2019; Prokin et al., 2020]. New for Kemerovo Area.

\section{Helochares obscurus (Müller, 1776)}

REFERENCES. Efomov, Zinchenko [2015].

MATERIAL EXAMINED. Kemerovo, small pool. 16.VIII.2009

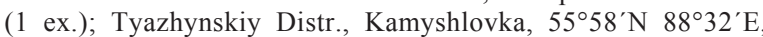
10.VI.2015, DE (1 ex.); Kemerovskiy Distr., Starochervovo, $55^{\circ} 10^{\prime} \mathrm{N} 86^{\circ} 22^{\prime}$ E, 22.VI.2015, 07.IX.2015, FB (17 exs.); Ke-

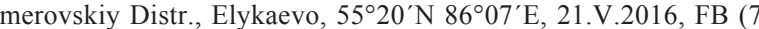
exs.); Krapivinskiy Distr., near Bannovo, $55^{\circ} 07^{\prime} \mathrm{N} 86^{\circ} 42^{\prime} \mathrm{E}, 14 . \mathrm{V}$. 2017, DE (1 ex.); Leninsk-Kuznetskiy Distr., vicinity of Podgor-

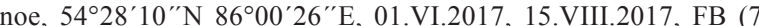
exs.); Chebulinskiy Distr., Chumay, Kiya River, 15.VII.2017, FB (1 ex.); Kemerovo, Krasnoe Lake, 25.VII.2017, FB (2 exs.), V. Zinchenko det.; Izhmorskiy Distr., Tunda, pond, 18.VIII.2018, DE (1 ex.); Yashkinskiy Distr., near Akatsiya, $55^{\circ} 42^{\prime} \mathrm{N} 85^{\circ} 29^{\prime} \mathrm{E}$, 20.VI.2018, DE (4 exs.)

DISTRIBUTION. Europe, Western Asia, Kazakhstan, Western Siberia, Western China [Fikáček et al., 2015; Przewoźny, 2019].

\section{Coelostoma orbiculare (Fabricius, 1775)}

\section{REFERENCES. Efimov [2010].}

MATERIAL EXAMINED. Kemerovo, small pool. 16.VIII.2009 (1 ex.); Krapivinskiy Distr., near "Azhendarovo", 20.-28.V.2012, 28.VII.2017, AK, DE (17 exs.); Leninsk-Kuznetskiy Distr., near

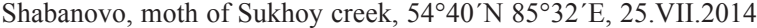
DE (1 ex.); Promyshlennovskiy Distr., Krasninskoe lake, 12.V.2015,

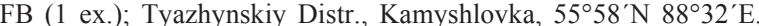
bog, 10.VI.2015, DE (2 exs.); Kemerovskiy Distr., Elykaevo, $55^{\circ} 20^{\prime} \mathrm{N}$ $86^{\circ} 07^{\prime}$ E, 21.V.2016, FB (1 ex.); Krapivinskiy Distr., vicinity of Zelenogorskiy, lake, $55^{\circ} 01^{\prime} \mathrm{N} 87^{\circ} 03^{\prime} \mathrm{E}$, 9.VII.2017, DE (1 ex.); Izhmorskiy Distr., Novyy Svet, shore of Zolotoy Kitat River, $55^{\circ} 46^{\prime} \mathrm{N}$ 86³9'E, 11.VII.2018, DE (1 ex.).

DISTRIBUTION. Europe, Turkey, Iran, Northern Asia, China [Fikáček et al., 2015; Przewoźny, 2019].

\section{Cercyon (Cercyon) bifenestratus Küster, 1851}

REFERENCES. Efimov [2010].

MATERIAL EXAMINED. Promyshlennovskiy Distr., Morozovo, pool, 22.VI.2012, DE (1 ex.), V. Zinchenko det.; Kemerovskiy Distr., Sukhovo, shore of Tom' River, 19.VII.2013, DE (2 exs.); Prokop'evskiy Distr., near Uskatskiy, Krivoy Uskat River, in water, 21.VIII.2014, DE (1 ex.), V. Zinchenko det.; Tyazhynskiy Distr.,

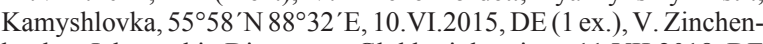
ko det.; Izhmorskiy Distr., near Glukharinka, river, 11.VII.2018, DE (2 exs.).

DISTRIBUTION. Europe, Northern Asia, Kazakhstan, Mongolia [Fikáček et al., 2015; Przewoźny, 2019].

\section{Cercyon (Cercyon)} convexiusculus Stephens, 1829

REFERENCES. Ryndevich [2003a].

MATERIAL EXAMINED. No specimens examined by authors. DISTRIBUTION. Europe, Western and Eastern Siberia [Fikáček et al., 2015; Przewoźny, 2019].

\section{Cercyon (Cercyon) lateralis (Marsham, 1802)}

REFERENCES. Ryndevich [2004]; Efimov [2010].

MATERIAL EXAMINED. Krapivinskiy Distr., near "Azhendarovo", 14.VII.2012, FB (1 ex.); Kemerovskiy Distr., Krekovo, 55³1'N 8552'E, 1.VII.2012, 22.VII.2012, 25.VIII.2013, 6.VI.2015, DE (7 exs.); Krapivinskiy Distr., Sheveli, dung, 14.V.2017, DE (1 ex.).

DISTRIBUTION. Europe, Canary Islands, Northern and Central Asia [Fikáček et al., 2015; Przewoźny, 2019], Canada, USA (introduced) [Hansen, 1999].
39. Cercyon (Cercyon) marinus Thomson, 1853

REFERENCES. Efimov [2010].

MATERIAL EXAMINED. Leninsk-Kuznetskiy Distr., $2 \mathrm{~km}$ E of Krasnoe vill., solonchak, near water, 54 $37^{\prime} \mathrm{N} 85^{\circ} 23^{\prime} \mathrm{E}$, 25.VII.2014, DE (1 ex.); Prokop'evskiy Distr., near Uskatskiy, Krivoy Uskat River, in water, 21.VIII.2014, DE (1 ex.), V. Zinchenko det.

DISTRIBUTION. Europe, Turkey, Northern and Central Asia, Mongolia, China, Japan [Fikáček et al., 2015; Przewoźny, 2019], Canada, USA [Hansen, 1999].

40. Cercyon (Cercyon) pygmaeus (Illiger, 1801)*

MATERIAL EXAMINED. Krapivinskiy Distr., Sheveli, dung, 14.V.2017, DE (1 ex.).

DISTRIBUTION. Europe, Egypt, Western and Eastern Siberia, Iran [Fikáček et al., 2015; Przewoźny, 2019], Canada, USA (introduced) [Hansen, 1999]. New for Kemerovo Area.

41. Cercyon (Cercyon) verus Shatrovskiy, 1989

REFERENCES. Ryndevich [2003a].

MATERIAL EXAMINED. No specimens examined by authors. DISTRIBUTION. ?Western Siberia (Kemerovo Area), Russian Far East (Kunashir) [Ryndevich et al., 2017].

REMARKS. The species is recorded for the Kemerovo Area for alone female. His occurrence here requires confirmation [Ryndevich et al., 2017].

\section{Cercyon (Cercyon) quisquilius (Linnaeus, 1760)}

REFERENCES. Ryndevich et al. [2017].

MATERIAL EXAMINED. No specimens examined by authors. DISTRIBUTION. Species with Polyzonal distribution [Hansen, 1999; Fikáček et al., 2015; Przewoźny, 2019].

43. Cercyon (Cercyon) unipunctatus (Linnaeus, 1758) REFERENCES. Ryndevich et al. [2017].

MATERIAL EXAMINED. No specimens examines by authors. DISTRIBUTION. Europe, Northern Asia, Northern China [Fikáček et al., 2015; Przewoźny, 2019], Canada, USA (introduced) [Hansen, 1999].

\section{Cercyon (Dicyrtocercyon) ustulatus (Preyssler, 1790)**}

Figs 2, 4, 6.

MATERIAL EXAMINED. Krapivinskiy Distr., "Azhendarovo",

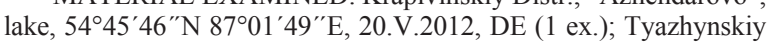

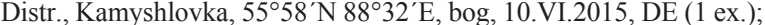
Izhmorskiy Distr., near Glukharinka, river, 11.VII.2018, DE (2 exs.).

DISTRIBUTION. Europe, Turkey, Iran [Fikáček et al., 2015; Przewoźny, 2019], Canada, USA (introduced) [Hansen, 1999]. Here this species is reliably recorded from Western Siberia for the first time.

REMARKS. An earlier reports of the species for Siberia [Zaitsev, 1908] has not yet been supported by factual material [Ryndevich, 2008]. Siberia has been excluded from the species range in new catalogs [e.g. Fikáček et al., 2015].

\section{Cercyon (Paracercyon) analis (Paykull, 1798)}

REFERENCES. Efimov [2010].

MATERIAL EXAMINED. Kemerovskiy Distr., Krekovo, $55^{\circ} 31^{\prime} \mathrm{N}$ $85^{\circ} 52^{\prime} \mathrm{E}, 12$.VII.2015, DE (1 ex.); vicinity of "Azhendarovo", moth of Bugas River, $54^{\circ} 44^{\prime} \mathrm{N} 87^{\circ} 01^{\prime} \mathrm{E}$, 10-30.VII.2008, AK (1 exs.).

DISTRIBUTION. Polyzonal distribution [Hansen, 1999; Fikáček et al., 2015; Przewoźny, 2019].

\section{Cercyon (Paracycreon) laminatus Sharp, 1873*}

MATERIAL EXAMINED. Kemerovo, at light, 01.VI.1998, AK (1 ex.), V. Zinchenko det.

DISTRIBUTION. Polyzonal distribution [Fikáček et al., 2015; Przewoźny, 2019]. New for Kemerovo Area. 


\section{Cryptopleurum crenatum (Kugelann, 1794)**}

Figs. 3, 5.

MATERIAL EXAMINED. Kemerovskiy Distr., Krekovo, $55^{\circ} 31^{\prime} \mathrm{N} 85^{\circ} 52^{\prime} \mathrm{E}, 22$.VII.2012, DE (3 exs.)

DISTRIBUTION. Europe, Turkey, Kazakhstan [Fikáček et al., 2015; Przewoźny, 2019]. New for Kemerovo Area and Asian part of Russia.

REMARKS. At least one find of the species was recorded in the USA on the east coast (Washington) [Smetana, 1978]. However, $C$. crenatum, unlike the other two species of the genus mentioned here, was not noted as an introducer anywhere. It is known from Asia only in two specimens: from Turkey (Trabzon) [Mart, 2009] and Kazakhstan (the vicinity of Almaty) [Prokin, 2010].

\section{Cryptopleurum minutum (Fabricius, 1775)*}

MATERIAL EXAMINED. Kemerovskiy Distr., Krekovo, $55^{\circ} 31^{\prime} \mathrm{N} 85^{\circ} 52^{\prime} \mathrm{E}, 22$.VII.2012, DE (4 exs.); Belovskiy Distr., vicinity of Novyy Gorodok, Bachatskie Sopki, 16.VIII.2014, DE (1 ex.).

DISTRIBUTION. Europe, Northern and Central Asia, China [Fikáček et al., 2015; Przewoźny, 2019], Canada, USA (introduced) [Hansen, 1999]. New for Kemerovo Area.

49. Cryptopleurum subtile Sharp, 1884

REFERENCES. Efimov [2010].

MATERIAL EXAMINED. No specimens examined by authors.

DISTRIBUTION. Polyzonal distribution [Hansen, 1999; Fikáček et al., 2015; Przewoźny, 2019].

- Megasternum concinnum (Marsham, 1802)

REMARKS. Findings previously published as M. concinnum [Hebauer, Ryndevich, 2005] are currently classified as M. immaculatum (Stephens, 1829) [Ryndevich, 2017].

50. Megasternum immaculatum (Stephens, 1829) REFERENCES. Ryndevich [2017].

MATERIAL EXAMINED. No specimens examined by authors.

DISTRIBUTION. Bulgaria, Great Britain, Poland, Western and Eastern Siberia [Ryndevich, 2017; Przewoźny, 2019].

REMARKS. See comment to previous species.

51. Pachysternum haemorrhoum Motschulsky, 1866

REFERENCES. Hebauer, Ryndevich [2005]; Efimov [2010].

MATERIAL EXAMINED. Kemerovskiy Distr., Krekovo, $55^{\circ} 31^{\prime} \mathrm{N} 85^{\circ} 52^{\prime} \mathrm{E}, 29 . \mathrm{V} .2011$, 1.VII.2012, DE (4 exs.); Krapivinskiy Distr., near “Azhendarovo", 14.VII.2012, FB (1 ex.); Chebulinskiy Distr., near Shestakovo, 9.VI.2015, DE (3 exs.).

DISTRIBUTION. Northern Asia, Kazakhstan, Mongolia, China, Korea, Japan [Fikáček et al., 2015; Przewoźny, 2019].

52. Sphaeridium bipustulatum Fabricius, 1781*

MATERIAL EXAMINED. Yashkinskiy Distr., Pacha, Tom' River flood-plane, 20.VII.2017, DE (3 exs.).

DISTRIBUTION. Holarctic [Przewoźny, 2019]. New for Kemerovo Area.

\section{Sphaeridium lunatum Fabricius, 1792}

REFERENCES. Efimov [2010].

MATERIAL EXAMINED. Krapivinskiy Distr., near "Azhendarovo", 10-30.VII.2008, 19-30.VI.2010, AK (2 exs.), V. Zinchen-

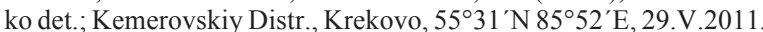
21.VI.2011, 01.VII.2012, DE (3 exs.); Yayskiy Distr., Beket, $56^{\circ} 22^{\prime} \mathrm{N}$ $86^{\circ} 28^{\prime} \mathrm{E}, 15$.VI.2018, DE (2 exs.).

DISTRIBUTION. Holarctic [Przewoźny, 2019].
54. Sphaeridium marginatum Fabricius, 1787

REFERENCES. Ryndevich [2003a]; Efimov, Zinchenko [2015]. MATERIAL EXAMINED. Yashkinskiy Distr., Pacha, Tom' River flood-plane, 20.VII.2017, DE (1 ex.).

DISTRIBUTION. Holarctic [Przewoźny, 2019].

55. Sphaeridium scarabaeoides (Linnaeus, 1758) REFERENCES. Efimov [2010].

MATERIAL EXAMINED. Kuznetskiy Alatau, Tisul'skiy Distr., Novyy Berikul', dung, 24.VII.1981, V.I. Eryshov leg. (1 ex.); Kemerovskiy Distr., near Podyakovo, 10.VII.1992, V. Ivanchikhin, Yu. Kabanenko (1 ex.), V. Zinchenko det.; Yashkinskiy Distr., Pacha, Tom' River flood-plane, 20.VII.2017, DE (10 exs.). 2019].

DISTRIBUTION. Polyzonal distribution [Przewoźny,

\section{Results}

Thus, 11 species of Helophoridae, 3 species of Hydrochidae and 41 species of Hydrophilidae were found on the territory of Kemerovo Area. In this checklist 31 species of Hydrophiloidea are recorded for the Kemerovo Area for the first time.

Helophorus discrepans and Cryptopleurum crenatum are recorded for the first time for the Asian part of Russia. Helophorus aspericollis and Enochrus melanocephalus are recorded for the first time for the Western Siberia.

Occurrence of species Cercyon ustulatus in Western Siberia is confirmed. Hydrophilus piceus, Hydrochara dichroma and Megasternum concinnum are excluded from the faunal checklist.

In the future research the faunal list of hydrophyloid beetles in the region will certainly be updated and supplemented. There is a high probability of finding here representatives of the Georissidae family, two species of which are known from Western Siberia [Przewoźny, 2019], and also widespread Palaearctic species Spercheus emarginatus (Schaller, 1783) of Spercheidae.

Acknowledgements. Authors are grateful to A.V. Korshunov, A.E. Kostyunin, V.A. Polevod, D.V. Sushchev, V. Akaev (Kemerovo) for providing the collection material, A.A. Prokin, A.S. Sazhnev (Borok) and V.K. Zinchenko (Novosibirsk) for valuable additional material. Special thanks go to R.B. Angus (London) for checking the definition of Helophorus aspericollis from photographs.

The study was supported by the Russian Foundation for Basic Research, project № 20-44-420008.

\section{References}

Berlov E.Ya. 1978. [Water scavenger beetles (Coleoptera, Hydrophilidae) of Irkutsk Area] // Nasekomye vostochnoy Sibiri. Irkutsk. P.65-72 [in Russian].

Efimov D.A. 2010. [To the knowledge of the fauna of hydrophilid beetles (Coleoptera: Hydrophilidae) of Kemerovo Region] // Caucasian Entomological Bulletin. Vol.6. No.1. P.23-24 [in Russian].

Efimov D.A., Zinchenko V.K. 2015. [New records of beetles (Coleoptera) in the fauna of Kemerovo Area] // Amurian Zoological Journal. Vol.7. No.3. P.223-226 [in Russian].

Fikáček M., Angus R.B., Gentili E., Jia F., Minoshima Y.N., Prokin A.A., Przewoźny M., Ryndevich S.K. 2015. Helophoridae Leach, 1815, Hydrochidae Thomson, 1859, Hydrophilidae Latreille, 
1802 // I. Löbl, D. Löbl (eds.). Catalogue of Palaearctic Coleoptera. Vol.2/1. Hydrophiloidea Staphylinoidea. Revised and Updated Edition. Leiden, Doston: Brill. P.25-33, 35-36, 37-76

Fossen E.I., Ekrem T., Nilsson A.N., Bergsten J. 2016. Species delimitation in northern European water scavenger beetles of the genus Hydrobius (Coleoptera, Hydrophilidae) // ZooKeys. Vol.564. P.71-120.

Hansen M. 1999. Hydrophiloidea (s.str.) (Coleoptera) // World Catalogue of Insects. Vol.2. Stenstrup: Appolo Books. 416 pp.

Hansen M. 2004. Family Helophoridae Leach, 1815 // I. Löbl, A Smetana (eds.). Catalogue of Palaearctic Coleoptera. Vol.2. Hydrophiloidea, Histeroidea, Staphylinoidea. Stenstrup: Apollo Books. P.36-41.

Hebauer F., Ryndevich S.K. 2005. New data on the distribution of the Old World Hydrophilidae (Coleoptera) // Acta Coleopterologica. Vol.11. No.1. P.43-51.

Mart A. 2009. Water scavenger beetles (Coleoptera: Hydrophilidae) provinces of Central Black Sea region of Turkey // Journal of the Entomological Research Society. Vol.11. No.1. P.47-70.

Prokin A.A. 2010. [New data on the distribution of Hydrochidae and Hydrophilidae (Coleoptera) in Russia and adjacent countries] // Problemy vodnoy entomologii Rossii i sopredel'nykh stran: Materialy X Trikhopterologicheskogo simpoziuma i IV Vserossiiskogo simpoziuma po amfibioticheskim i vodnym nasekomym (Vladikavkaz, Russia, 5-7 May 2010). Vladikavkaz: North Ossetian State University Publ. P.74-78 [in Russian].

Prokin A.A., Chuluunbaatar G., Angus R.B., Jäch M.A., Petrov P.N., Ryndevich S.K., Byambanyam E., Sazhnev A.S., Hájek J., Shaverdo H. 2020. New records of water beetles (Coleoptera Gyrinidae, Haliplidae, Noteridae, Dytiscidae, Helophoridae, Hydrophilidae, Hydraenidae) and shore beetles (Coleoptera: Heteroceridae) of Mongolia // Aquatic Insects. Vol.41. No.1. P.1-44. DOI: 10.1080/01650424.2019.1651870.

Prokin A.A., Ryndevich S.K., Petrov P.N., Andrejeva T.R. 2008. New data on the distribution of Helophoridae, Hydrochidae and Hydrophilidae (Coleoptera) in Russia and adjacent lands // Russian Entomological Journal. Vol.17. No.2. P.1-4.

Przewoźny M. 2019. Catalogue of Palearctic Hydrophiloidea (Coleoptera). Internet version 2019.01.01. http://www.waterbeetles.eu/ documents/PAL_CAT_Hydrophiloidea_2019.pdf

Ryndevich S.K. 2003a. Some records of Dytiscidae, Helophoridae, Hydrochidae, Hydrophilidae and Hydraenidae in Russia and other regions (Coleoptera) // Latissimus. No.16. P.17-20.

Ryndevich S.K. 2003b. A review of the genus Anacaena Thomson, 1859 for the European part of Russia and adjacent regions (Coleoptera, Hydrophilidae) // Eurasian Entomological Journal. Vol.2. No.4. P.265-274.

Ryndevich S.K. 2004. Review of species of the genus Cercyon Leach, 1817 of Russia and adjacent regions. I. Subgenus Cercyon (s.str.) Leach, 1817. Cercyon lateralis-group (Coleoptera: Hydrophilidae) // Annales Universitatis Mariae Curie-Sklodowska (Section C). Vol.59. P.1-13.

Ryndevich S.K. 2008. Review of species of the genus Cercyon Leach, 1817 of Russia and adjacent regions. IV. The subgenra Paracycreon Orchymont, 1942 and Dicyrtocercyon Ganglbauer, 1904 (Coleoptera: Hydrophilidae) // Zoosystematica Rossica. Vol.17. No.2. P.89-97.

Ryndevich S.K. 2014. [The fauna of hydrophiloids (Coleoptera: Hydrophiloidea) in the subtaiga zone of the Palaearctic] // BarSU Herald. Series of biological sciences (general biology), agricultural sciences (agronomy). Vol.2. P.19-35 [in Russian].

Ryndevich S.K. 2017. New faunistic records of hydrophilid beetles (Coleoptera: Hydrophiloidea: Hydrophilidae) from Eurasia // BarSU Herald. Series of biological sciences (general biology), agricultural sciences (agronomy). Vol.5. P.65-70.

Ryndevich S.K., Jia F., Fikáček M. 2017. A review of the Asian species of the Cercyon unipunctatus group (Coleoptera: Hydrophilidae: Sphaeridiinae) // Acta Entomologica Musei Nationalis Pragae. Vol.57. No.2. P.535-576.

Shaarawi F.A., Angus R.B. 1990. A chromosomal investigation of five European species of Anacaena Thomson (Coleoptera: Hydrophilidae) // Entomologica Scandinavica. Vol.21. P.415426.

Short A.E. 2017. Systematics of aquatic beetles (Coleoptera): current state and future directions // Systematic Entomology. Vol.43. No.1. P.1-18.

Smetana A. 1978. Revision of the subfamily Sphaeridiinae of America north of Mexico (Coleoptera: Hydrophilidae) // Memoirs of the Entomological Society of Canada. Vol.110. No.105. P.1-292.

Zaitsev F.A. 1908. Catalogue des Coleopteres aquatiques des familles des Dryopidae, Georyssidae, Cyathoceridae, Heteroceridae et Hydrophilidae // Horae Societatis Entomologica Rossicae. Vol.38. No.4. P.283-420. 